Short Communication

\title{
High catalytic activity of indium tin oxide nanoparticle modified electrode towards electro-oxidation of ascorbic acid
}

\author{
Ming-Yuan Wei ${ }^{1}$, Rongfu Huang, Liang-Hong Guo * \\ State Key Laboratory of Environmental Chemistry and Ecotoxicology, Research Center for Eco-environmental Sciences, Chinese Academy of Sciences, P.O. Box 2871, 18 \\ Shuangqing Road, Beijing 100085, China
}

\section{A R T I C L E I N F O}

\section{Article history:}

Received 11 July 2011

Received in revised form 30 September

2011

Accepted 26 October 2011

Available online 4 November 2011

\section{Keywords:}

Electrocatalysis

Metal oxide nanoparticle

Ascorbic acid

Oxygen vacancy defect

\begin{abstract}
A B S T R A C T
Metal nanoparticles and carbon nanotubes have been shown to possess high electrocatalytic activity. Indium tin oxide (ITO) is a popular electrode material, but the electro-catalytic properties of its nanomaterials have not been reported. We demonstrate here for the first time facile electrocatalytic oxidation of ascorbic acid on ITO nanoparticle-modified electrodes. Compared to the conventional ITO thin film electrode, the voltammetric peak potential for ascorbic acid oxidation was lowered by $800 \mathrm{mV}$ on ITO nanoparticle-modified electrodes to a potential similar to metal electrodes. The ITO nanoparticle was composed of $90 \% \mathrm{In}_{2} \mathrm{O}_{3}$ and $10 \% \mathrm{SnO}_{2}$. Since the electrocatalytic activity was also found on $\operatorname{In}_{2} \mathrm{O}_{3}$ nanoparticle electrodes but not on $\mathrm{SnO}_{2}$ nanoparticle electrodes, the $\mathrm{In}_{2} \mathrm{O}_{3}$ composition in ITO nanoparticle is mainly responsible for the high activity. In photoluminescence measurement, two intense emission peaks at $415 \mathrm{~nm}$ and $438 \mathrm{~nm}$ associated with surface oxygen vacancies were observed on the semiconductor electrodes. It was hypothesized that the oxygen vacancies could be the active sites for electrocatalytic reactions. A linear relationship between the oxidation current and ascorbic acid concentration was found in the range of $10 \mu \mathrm{M}$ to $5 \mathrm{mM}$, with a lower detection limit of $5 \mu \mathrm{M}$ and $7.9 \% \mathrm{RSD}(n=11)$. The high electro-catalytic activity and transmittance of $\mathrm{In}_{2} \mathrm{O}_{3}$ and ITO nanoparticle electrodes make them potentially very useful in opto-electronic devices and chemical/bio-sensors.
\end{abstract}

(c) 2011 Elsevier B.V. All rights reserved.

\section{Introduction}

Nanomaterials have found wide applications in fundamental electrochemical research, electrochemical sensors and biosensors, and energy storage and conversion devices due to their unique physical, electronic, and chemical properties that are distinctively different from the corresponding bulk materials. Some nanomaterials, mostly metal nanoparticles, possess excellent catalytic properties towards the redox reaction of endogenous chemicals such as dopamine, ascorbic acid, NADH, glucose, $\mathrm{H}_{2} \mathrm{O}_{2}$ and NO, which play important roles in biological processes. On nanomaterial-based electrodes, the redox reaction was greatly enhanced, displaying reduced overpotential and improved reversibility [1-3]. Among the studies of gold and platinum nanoparticles deposited on conventional electrodes, a few of them compared directly with polycrystalline surface and demonstrated that the catalysis could be attributed to the nanometer size and structure [4-7]. Electrocatalytic oxidation of dopamine, NADH and ascorbic acid was also

\footnotetext{
* Corresponding author. Tel./fax: +86 1062849685.

E-mail address: LHGuo@rcees.ac.cn (L.-H. Guo)

1 Present address: Lane Department of Computer Science and Electrical Engineering, College of Engineering and Mineral Resources, WV Nano Initiative, West Virginia University, Morgantown, WV 26506-6109, USA.
}

reported on carbon nanotube modified electrodes [8-10]. Careful studies suggest that the catalysis most likely occurred at the graphitic edge-plane sites at the exposed ends of the nanotube, similar to conventional graphite electrodes [11-14].

Thanks to its unique characteristics of high optical transmittance, good electrical conductivity and wide potential window, indium tin oxide (ITO) thin film electrode is widely used in optoelectronic devices as well as electrochemical (bio)sensors. Electrochemical reaction of many organic molecules such as dopamine [15], ascorbic acid [16], tripropal amine [17] and oxalic acid [18] on ITO is significantly slower than metal and carbon electrodes, likely due to the lack of catalytic sites on ITO. Specifically, ascorbic acid exhibits an apparent electrooxidation response at $0.5 \mathrm{~V}$ on metal electrodes $[19,20]$ and at $0.6 \mathrm{~V}$ on glassy carbon electrode [21], whereas the oxidation reaction is inhibited and thereby results in a large overpotential on ITO [16]. ITO and other metal oxide semiconductor nanomaterials may provide enhanced electro-catalytic properties, but few studies have been reported in this area. In our recent work, ITO nanoparticle modified electrodes were found to catalyze the electrochemical oxidation of tripropyl amine (TPA) and enhance electrochemiluminescence [22]. However, the catalytic mechanism was difficult to investigate due to the overlap of TPA oxidation wave with that of water. Herein, we demonstrate for the first time facile electrocatalytic oxidation of 
ascorbic acid on metal oxide nanoparticle modified electrodes at a potential similar to metal electrodes. The mechanism for the enhanced electrocatalytic activity is also proposed.

\section{Experimental}

\subsection{Materials and reagents}

Poly(vinyl alcohol) (PVA, fully hydrolyzed) was obtained from Sigma-Aldrich (St. Louis, MO, USA). Ascorbic acid was purchased from Beijing Chemicals Corporation (Beijing, China). All other chemical reagents were of analytical reagent (AR) grade. Solutions were prepared in high-purity water from a Millipore milli-Q (Biocel) water purification system (Billerica, MA, USA). Ascorbic acid was dissolved in phosphate buffered saline (PBS, containing $137 \mathrm{mM} \mathrm{NaCl}, 2.7 \mathrm{mM} \mathrm{KCl}, 10 \mathrm{mM} \mathrm{Na}_{2} \mathrm{HPO}_{4}$ and $1.5 \mathrm{mM} \mathrm{KH}_{2} \mathrm{PO}_{4}$, $\mathrm{pH} 7.3)$.

\subsection{Preparation of ITO nanoparticle electrode}

Radio-frequency sputtered ITO conductive glass (film thickness: $900 \pm 100 \AA$, conductivity: $19 \pm 2.1 \Omega / \square$ ) was obtained from Nanbo Corporation (Shenzhen, Guangdong, China), and was cut into $5 \mathrm{~cm} \times 5 \mathrm{~cm}$ size slices. The slices were ultrasonically cleaned according to the published procedure [23], dried in an oven at $50{ }^{\circ} \mathrm{C}$, followed by treating for $60 \mathrm{~s}$ with an Atmospheric Plasma Surface Treatment Systems CTD-1000z from Kulun Electronics Co., Ltd. (Nanjing, Jiangsu, China). ITO nanoparticle solution (10 wt\%, with a primary particle size of $20-30 \mathrm{~nm}$ ) was purchased from Shanghai Huzheng Nanotechnology Co., Ltd. (Shanghai, China). $10 \mu \mathrm{L}$ Tween-20 was added to $10 \mathrm{~mL}$ ITO nanoparticle solution, and stirred for 30 more minutes. The solution was spread on the ITO slices by spin-coating $\left(200 \mathrm{rmin}^{-1}\right.$ for $10 \mathrm{~s}$ and then $1500 \mathrm{r} \mathrm{min}^{-1}$ for $50 \mathrm{~s}$ ) using a transparent tape to define the area and thickness, and was dried in air on a warm plate. The film was annealed at $450{ }^{\circ} \mathrm{C}$ for $30 \mathrm{~min}$. The ITO nanoparticle-modified glass was cut into $2.5 \mathrm{~cm} \times 0.5 \mathrm{~cm}$ electrodes, washed with purified water, and dried under a stream of nitrogen before electrochemical measurement.

\subsection{Preparation of tin oxide and indium oxide nanoparticle electrode}

Tin oxide ( $15 \mathrm{wt} \%$ in $\mathrm{H}_{2} \mathrm{O}$ colloidal dispersion, particle size: 15-20 nm) and indium oxide nanoparticle powder (particle size: $\sim 50 \mathrm{~nm}$ ) were purchased from Alfa Aesar (Ward Hill, MA, USA). Indium oxide nanoparticles were first dispersed ultrasonically with PVA, thus an indium oxide nanoparticle colloid was obtained. $\mathrm{SnO}_{2}$ and $\mathrm{In}_{2} \mathrm{O}_{3}$ nanoparticle modified electrodes were prepared from the colloids by following the same procedure as for ITO nanoparticles.

\subsection{SEM characterization}

Electrode surface was characterized by a JSM-6700F field emission scanning electron microscope (JEOL Ltd., Tokyo, Japan).

\subsection{Electrochemical measurement}

Cyclic voltammetry was performed on a CHI 400A electrochemistry analyzer from $\mathrm{CH}$ Instruments (Austin, TX, USA) with a Pt counter electrode and an $\mathrm{Ag} / \mathrm{AgCl}(3 \mathrm{M} \mathrm{KCl})$ reference electrode (also from $\mathrm{CHI}$ ). The electrode area in contact with the electrolyte was $25 \mathrm{~mm}^{2}$.

\subsection{Photoluminescence (PL) measurement}

PL emission was measured on a PerkinElmer luminescence spectrometer LS-55 (Waltham, MA, USA) with the front surface accessory. Excitation: $380 \mathrm{~nm}$; slit width: $5 \mathrm{~nm}$; filter: $350 \mathrm{~nm}$ cut-off; emission scan from $400 \mathrm{~nm}$ to $600 \mathrm{~nm}$.

\subsection{X-ray photoelectron spectroscopy measurement}

XPS measurements were made on a VG ESCALAB MkII spectrometer (Thermo VG Scientific, West Sussex, UK) with a Mg Ka $\mathrm{X}$-ray source ( $1253.6 \mathrm{eV}$ photons). All binding energies (BEs) were referenced to the neutral $C_{1 s}$ peak at $284.6 \mathrm{eV}$.

\section{Results and discussion}

ITO nanoparticle-modified electrodes were fabricated on sputtered ITO glass from a commercially available colloidal solution of dispersed nanoparticles by following the procedure described previously $[24,25]$. As shown in the scanning electron micrographs (Fig. 1), the sputtered ITO film was found to be quite flat, composed mostly of large terraces. By comparison, the nanoparticle surface was much rougher, exhibiting grooves and pores of various sizes. Individual particles of $20-30 \mathrm{~nm}$ diameter were clearly visible, and were within the range specified by the manufacturer. To quantify the increase in surface area of the nanoparticle-modified electrode, cyclic voltammetry of both unmodified and modified ITO electrodes was preformed in a phosphate buffer. As shown in Fig. 2, the capacitive current of the nanoparticle electrode measured from the cyclic voltammogram was approximately 10 times larger than the unmodified ITO. The increase in capacitance is consistent with the increased surface roughness observed in the SEM image, and also suggests the nanoparticles deposited on ITO glass are electronically conductive.

It has been found that, in general, electrochemical reaction of many organic molecules such as dopamine [15], ascorbic acid [16], tripropal amine [17] and oxalic acid [18] on ITO is significantly slower than metal and carbon electrodes. This has been attributed to the lack of catalytic sites on ITO, although the exact mechanism is still not clear. Ascorbic acid is a naturally occurring organic compound in biological systems. It usually acts as an antioxidant to eliminate reactive oxygen species such as hydroxyl radicals which are damaging to nucleic acids, proteins, and lipids. Due to its redox reactivity, electrochemistry has become a popular method for the detection of ascorbic acid in biological fluids. As shown in Fig. 3, cyclic voltammetry of ascorbic acid on sputtered ITO electrode was very irreversible, depicting a broad oxidation wave with a peak potential at $c a$. $1.1 \mathrm{~V}$. However, after ITO nanoparticles were deposited on the same electrode, the voltammogram changed dramatically. The oxidation peak potential shifted negatively to $0.3 \mathrm{~V}$, which is close to the oxidation potential of ascorbic acid on activated glassy carbon electrode [21] and Au nanoparticles-modified electrodes [26]. By comparison, the sputtered ITO electrode did not exhibit such electrocatalytic activity even after annealing at $450^{\circ} \mathrm{C}$. In addition, X-ray photoelectron spectroscopy analysis revealed that no other elements had been introduced during the nanoparticle electrode preparation, and the intensity ratio of $\mathrm{In} / \mathrm{Sn}$ was the same on both types of electrodes (Fig. 2 inset). Since the ITO nanoparticle was composed of $90 \% \mathrm{In}_{2} \mathrm{O}_{3}$ and $10 \% \mathrm{SnO}_{2}$, the following experiments were carried out to identify which of the nanoparticle components was responsible for the enhanced activity. $\mathrm{In}_{2} \mathrm{O}_{3}$ and $\mathrm{SnO}_{2}$ nanoparticle-modified electrodes were separately prepared, characterized by SEM, and tested. In Fig. 1E and F, one can see that the two electrodes looked very similar to ITO nanoparticle electrodes in terms 

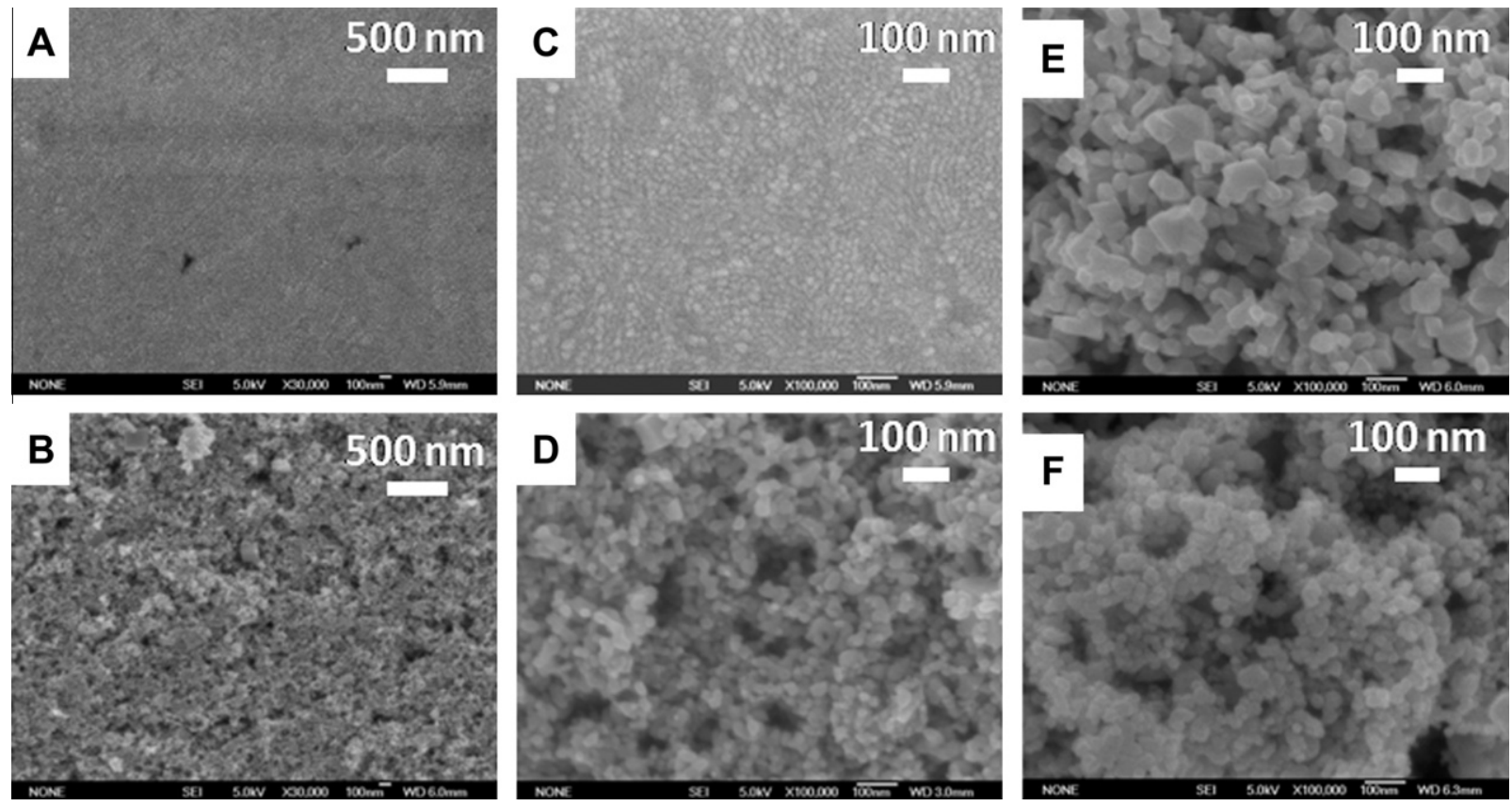

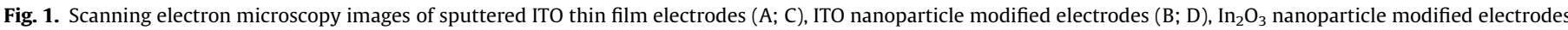
(E) and $\mathrm{SnO}_{2}$ nanoparticle modified electrodes (F). Magnification: 3000X (A; B), and 10000X (C; D; E; F).

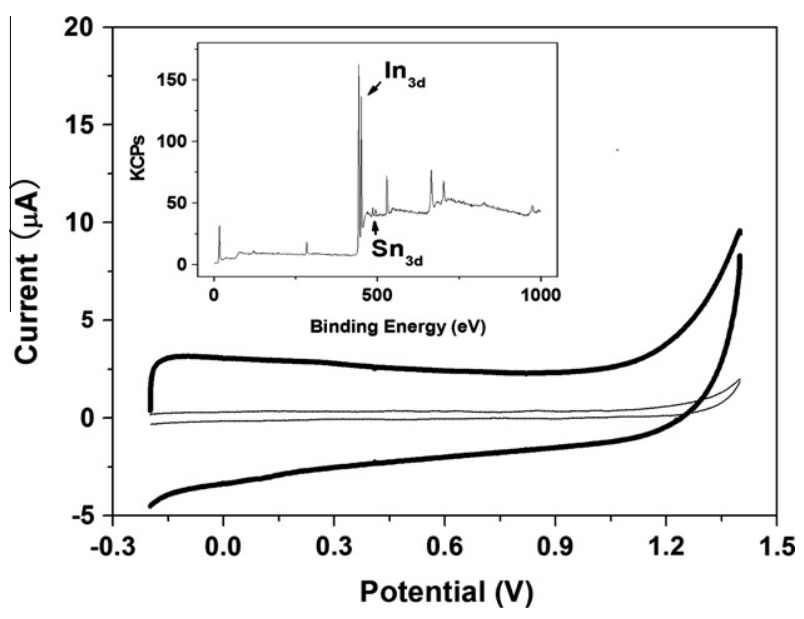

Fig. 2. Cyclic voltammograms of sputtered ITO thin film electrodes (thin line) and ITO nanoparticle modified electrodes (thick line) in PBS (pH 7.3). Inset: XPS spectra of ITO nanoparticle modified electrodes. The electrode area in contact with the electrolyte was $25 \mathrm{~mm}^{2}$. Potential scan rate: $50 \mathrm{mV} \mathrm{s}^{-1}$.

of surface morphology. In accordance with the particle size in the colloidal suspension, the $\operatorname{In}_{2} \mathrm{O}_{3}$ nanoparticle in the electrode film appeared much larger than $\mathrm{SnO}_{2}\left(50 \mathrm{~nm} \mathrm{In}_{2} \mathrm{O}_{3}\right.$ was the only size available to us). As shown also in Fig. 3, two remarkably different voltammograms were obtained in ascorbic acid. The response on $\mathrm{In}_{2} \mathrm{O}_{3}$ nanoparticle-modified electrode is very similar to the one on ITO nanoparticle electrode, whereas the voltammogram of $\mathrm{SnO}_{2}$ nanoparticle electrode resembles that of the sputtered ITO electrode. These results indicate that $\mathrm{In}_{2} \mathrm{O}_{3}$ in ITO nanoparticle is the major contributor to its electrocatalytic activity.

Like ascorbic acid, dopamine is also an analyte of biological importance. Cyclic voltammetry of dopamine on ITO electrodes was therefore conducted. As can be seen in Fig. 4, the ITO nanoparticle electrode exhibited only slight improvement in electro-oxidation of dopamine over the sputtered film electrode. This is probably

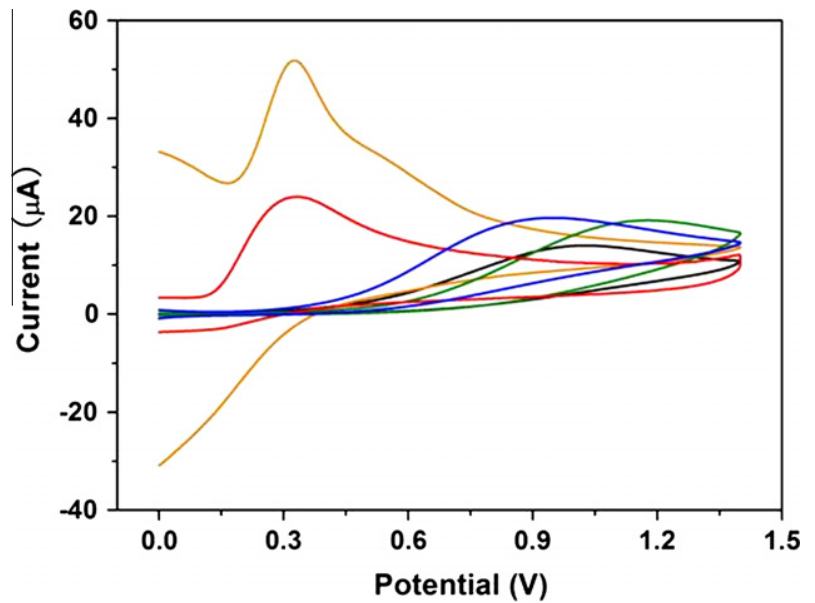

Fig. 3. Cyclic voltammograms of sputtered ITO thin film electrodes before (black) and after (green) annealing at $450^{\circ} \mathrm{C}$, ITO nanoparticle modified electrodes (red) $\mathrm{In}_{2} \mathrm{O}_{3}$ nanoparticle modified electrodes (orange) and $\mathrm{SnO}_{2}$ nanoparticle modified electrodes (blue) in $0.5 \mathrm{mM}$ ascorbic acid (PBS, pH 7.3). The electrode area in contact with the electrolyte was $25 \mathrm{~mm}^{2}$. Potential scan rate: $50 \mathrm{mV} \mathrm{s}^{-1}$. (For interpretation of the references to color in this figure legend, the reader is referred to the web version of this article.)

because the electrode reaction on the latter electrode is already quite fast.

Previous studies have found that ITO preparation method, its surface pretreatment and cleaning procedures all have profound impacts on the electrochemical activity of reversible probes such as ferrocene dicarboxylic acid and ferricyanide due to the existence of surface defects and adsorbates [27]. We hypothesize that the high electrocatalytic activity of $\mathrm{In}_{2} \mathrm{O}_{3}$ nanoparticle electrodes toward ascorbic acid oxidation could also be related to surface defects on the nanoparticle. It has been shown that oxygen vacancy defects in indium oxide crystals can be verified by photoluminescence measurement. Under the excitation of $380 \mathrm{~nm}$ irradiation, 


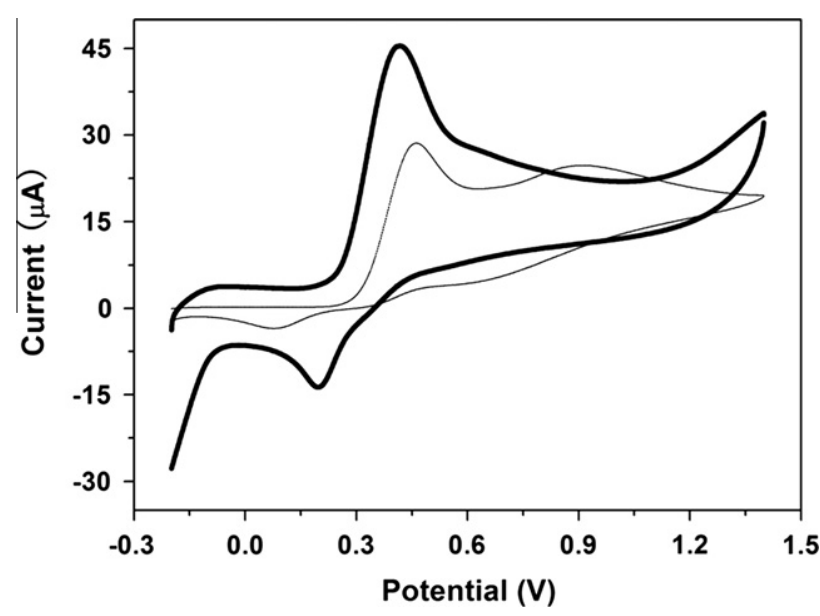

Fig. 4. Cyclic voltammograms of $0.5 \mathrm{mM}$ dopamine (PBS, $\mathrm{pH} 7.3$ ) on a sputtered ITO electrode (thin line), and ITO NP electrode (thick line). The electrode area in contact with the electrolyte was $25 \mathrm{~mm}^{2}$. Potential scan rate: $50 \mathrm{mV} \mathrm{s}^{-1}$.

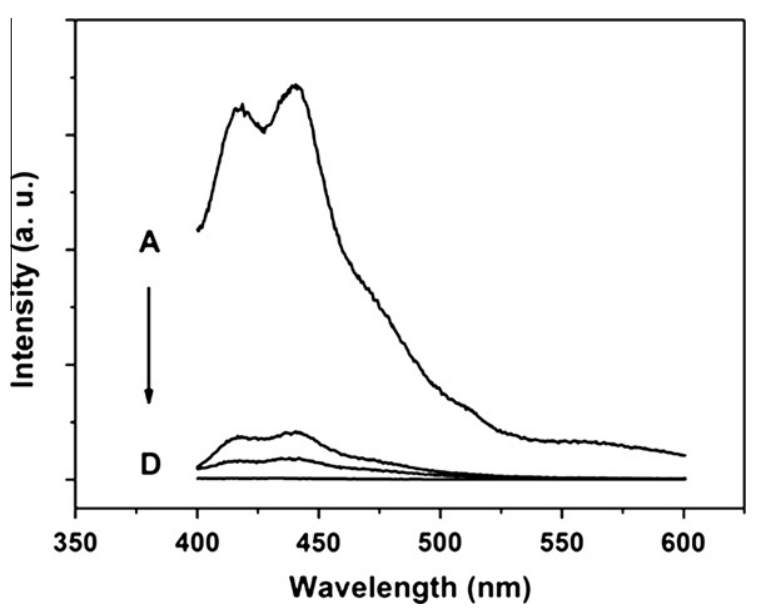

Fig. 5. Photoluminescence spectra of $(A) \mathrm{In}_{2} \mathrm{O}_{3}$ nanoparticle modified electrodes, (B) ITO nanoparticle modified electrodes, (C) sputtered ITO thin film electrodes, and (D) $\mathrm{SnO}_{2}$ nanoparticle modified electrodes.

two well-resolved emission peaks located at $415 \mathrm{~nm}$ and $438 \mathrm{~nm}$ were observed on an $\operatorname{In}_{2} \mathrm{O}_{3}$ nanoparticle electrode, as shown in Fig. 5. The two emission peaks have been assigned to different emission energy levels generated by oxygen vacancies [28]. The strong photoluminescence emission points to a large number of oxygen vacancies on $\mathrm{In}_{2} \mathrm{O}_{3}$ nanoparticle surface. By comparison, emission from $\mathrm{SnO}_{2}$ nanoparticle and sputtered ITO surface is much weaker. The data suggest the electrocatalytic activity observed with $\mathrm{In}_{2} \mathrm{O}_{3}$ nanoparticle electrodes could be attributed to the oxygen vacancies on the nanoparticle, although quantitatively the electrocatalytic current and photoluminescence intensity did not correlate well.

The analytical performance of the ITO nanoparticle electrode in the determination of ascorbic acid was assessed in the following experiments. As shown in Fig. 6, the peak oxidation current increased with increasing concentrations of ascorbic acid, and demonstrated a linear dependence in the range of $10 \mu \mathrm{M}$ to $5 \mathrm{mM}$ $\left(y=51.09 x+0.0315, R^{2} 0.9997\right)$. The lower limit of detection was calculated to be $5 \mu \mathrm{M}$ (S/B 3). With $0.5 \mathrm{mM}$ ascorbic acid as the analyte, the reproducibility of the nanoparticle electrodes was quite good, with an RSD of $7.9 \%(n=11)$.
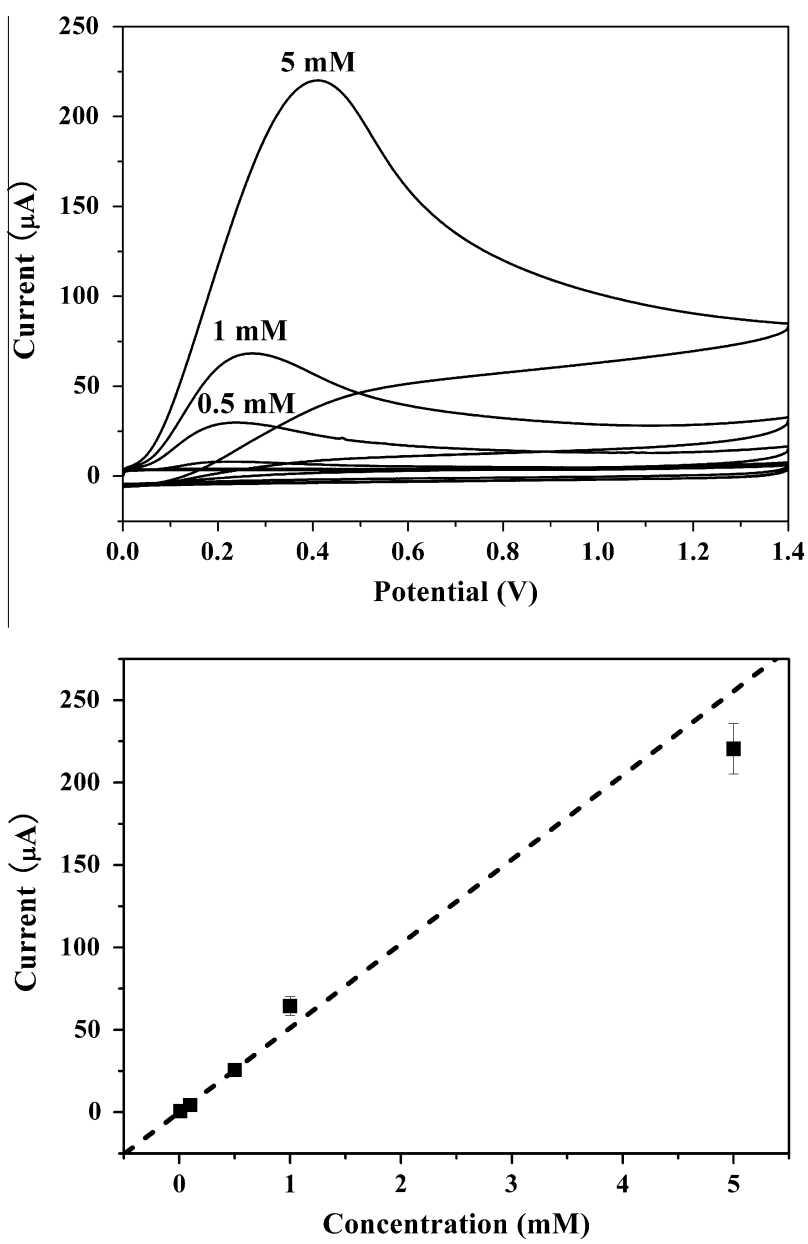

Fig. 6. (Top) Cyclic voltammograms of ITO nanoparticle modified electrodes in 0.01 , $0.1,0.5,1$ and $5 \mathrm{mM}$ ascorbic acid (PBS, pH 7.3). (Bottom) The linear plot of background subtracted anodic peak current versus the concentration of ascorbic acid $(n=6)$. The electrode area in contact with the electrolyte was $25 \mathrm{~mm}^{2}$. Potential scan rate: $50 \mathrm{mV} \mathrm{s}^{-1}$.

\section{Conclusions}

Facile electrocatalytic oxidation of ascorbic acid on indium tin oxide and indium oxide nanoparticle modified electrodes was achieved, which showed $800 \mathrm{mV}$ lower peak potential than the bulk ITO electrode and was attributed to the oxygen vacancy defects on $\operatorname{In}_{2} \mathrm{O}_{3}$ surface. Study is currently underway for more indepth investigation on the mechanisms of nanoparticle-enhanced electrocatalytic activity and its size dependence. Because of their high optical transmittance and ease of micro-fabrication, metal oxide nanoparticle electrodes are uniquely suited for miniaturized opto-electronic devices and chemical/bio-sensors.

\section{Acknowledgements}

The authors acknowledge the National Basic Research Program of China (2011CB936001) and the National Natural Science Foundation of China $(90813016,20825519,20921063)$ for financial support.

\section{References}

[1] X.L. Luo, A. Morrin, A.J. Killard, M.R. Smyth, Electroanalysis 18 (2006) 319-326.

[2] C. Welch, R. Compton, Anal. Bioanal. Chem. 384 (2006) 601-619.

[3] R.L. McCreery, Chem. Rev. 108 (2008) 2646-2687.

[4] M.S. El-Deab, T. Ohsaka, Electrochem. Commun. 4 (2002) 288-292. 
[5] C.R. Raj, A.I. Abdelrahman, T. Ohsaka, Electrochem. Commun. 7 (2005) 888893.

[6] L. Wang, J.Y. Bai, P.F. Huang, H.J. Wang, L.Y. Zhang, Y.Q. Zhao, Electrochem. Commun. 8 (2006) 1035-1040.

[7] B.K. Jena, C.R. Raj, J. Phys. Chem. C 111 (2007) 6228-6232.

[8] G.A. Rivas, M.D. Rubianes, M.C. Rodriguez, N.E. Ferreyra, G.L. Luque, M.L. Pedano, S.A. Miscoria, C. Parrado, Talanta 74 (2007) 291-307.

[9] M. Trojanowicz, Trac-Trend. Anal. Chem. 25 (2006) 480-489.

[10] D. Vairavapandian, P. Vichchulada, M.D. Lay, Anal. Chim. Acta 626 (2008) 119129.

[11] C.E. Banks, R.R. Moore, T.J. Davies, R.G. Compton, Chem. Commun. (2004) 1804-1805.

[12] C.E. Banks, T.J. Davies, G.G. Wildgoose, R.G. Compton, Chem. Commun. (2005) 829-841.

[13] A. Chou, T. Bocking, N.K. Singh, J.J. Gooding, Chem. Commun. 1 (2005) 842844.

[14] G.G. Wildgoose, C.E. Banks, H.C. Leventis, R.G. Compton, Microchim. Acta 152 (2006) $187-214$
[15] G. Xu, Y. Iwasaki, O. Niwa, Chem. Lett. 34 (2005) 1120-1122.

[16] D. Kato, G. Xu, Y. Iwasaki, Y. Hirata, R. Kurita, O. Niwa, Langmuir 23 (2007) 8400-8405.

[17] M.-T. Chiang, C.-W. Whang, J. Chrom. A 934 (2001) 59-66.

[18] X. Zhang, A.J. Bard, J. Phys. Chem. 92 (1988) 5566-5569.

[19] M. Rueda, A. Aldaz, F. Sanchez-Burgos, Elecrochim. Acta 23 (1978) 419-424.

[20] P. Kambinas, D. Jannakoudakes, J. Electroanal. Chem. 160 (1984) 159-167.

21] I.F. Hu, T. Kuwana, Anal. Chem. 58 (1986) 3235-3239.

[22] R. Huang, M.Y. Wei, L.H. Guo, J. Electroanal. Chem. 656 (2011) 136-139.

[23] L.H. Guo, M.Y. Wei, H. Chen, J. Phys. Chem. B 110 (2006) 20568-20571.

[24] M. Liang, S. Liu, M. Wei, L.H. Guo, Anal. Chem. 78 (2006) 621-623.

[25] X.Q. Yang, L.H. Guo, Anal. Chim. Acta 632 (2009) 15-20.

[26] J. Zhang, M. Oyama, Electrochem. Commun. 9 (2007) 459-464.

[27] C. Donley, D. Dunphy, D. Paine, C. Carter, K. Nebesny, P. Lee, D. Alloway, N.R. Armstrong, Langmuir 18 (2002) 450-457.

[28] J. Yang, C. Lin, Z. Wang, J. Lin, Inorg. Chem. 45 (2006) 8973-8979. 